\title{
CORRIGENDA
}

\section{Prostatic abscess due to Candida tropicalis}

\author{
C Bastide, A Carcenac, F Arrova and D Rossi \\ Prostate Cancer and Prostatic Diseases (2009) 12, 382; doi:10.1038/pcan.2009.40
}

Correction to: Prostate Cancer and Prostatic Diseases (2005) 8, 296-297. doi:10.1038/sj.pcan.4500809

Since the publication of the above paper, the authors have identified that the contributing author's last name,
F Arrova, was misspelled. The corrected author's name should read:

F Arroua

\section{Awareness of prostate cancer among patients and the general public: results of an international survey}

\author{
JM Fitzpatrick, RS Kirby, CL Brough and AL Saggerson
}

Prostate Cancer and Prostatic Diseases (2009) 12, 382; doi:10.1038/pcan.2009.47

Correction to: Prostate Cancer and Prostatic Diseases (this issue), published online 21 July 2009; doi:10.1038/ pcan. 2009.30

The figure legends as published do not appropriately indicate specific areas of the figures.

HTML (full-text) version of the article:

In the legend to Figure 3, the colors were incorrectly attributed in the following description:

'Green shading: very low risk; pink shading: low risk; navy shading: quite low risk; yellow shading: very high risk.'

The legend should read as follows:

Figure 3 Perceptions of prostate cancer risk among respondents (split by sample). Green shading: very low risk; yellow shading: low risk; pink shading: quite high risk; navy shading: very high risk. The color reproduction of this figure is available in the html full-text version of the paper.

\section{PDF and print versions of the article:}

The legends incorrectly refer to colors in the figure, but the figures appear in black and white in the PDF and print versions of the article. The legends should read as follows:

Figure 1 Perceptions of respondents (grouped by sample) when asked if they thought prostate cancer or breast cancer was more common in their country. Lefthand bars: breast cancer is more common; right-hand bars: prostate cancer is more common. The color reproduction of this figure is available in the html fulltext version of the paper.

Figure 2 Responses of participants (well sample) when they were asked 'who does prostate cancer affect: men, women or both?' Very light shading: don't know; light shading: only men; medium shading: only women; dark shading: both. The color reproduction of this figure is available in the html full-text version of the paper.

Figure 3 Perceptions of prostate cancer risk among respondents (split by sample). Far left bar: very low risk; middle left: low risk; middle right: quite high risk; far right: very high risk. The color reproduction of this figure is available in the html full-text version of the paper.

Figure 4 Reasons cited for perceived low prostate cancer risk among those who answered 'low' to the previous question (split by sample). Light bars: prostate cancer sample; dark bars: well sample. The color reproduction of this figure is available in the html full-text version of the paper. 